\title{
Florística e fitossociologia em áreas de Savana Estépica Parque em Barra do Quaraí-RS, Brasil
}

\author{
Floral and phytosociology in Park steppe savannah areas in Barra of Quaraí-RS, \\ Brazil
}

\author{
Luciano Farinha Watzlawick ${ }^{1 *}$, Edson Gardin² e Joelmir Augustinho Mazon ${ }^{3}$ \\ ${ }^{1}$ Departamento de Agronomia da Universidade Estadual do Centro-Oeste do Paraná (UNICENTRO), Paraná, Brasil. \\ ${ }^{2}$ Doutorando em Produção Vegetal do Programa de Pós-Graduação em Agronomia da Universidade Estadual do \\ Centro-Oeste do Paraná (UNICENTRO), Paraná, Brasil. \\ ${ }^{3}$ Mestre em Ciências Florestais pela Universidade Estadual do Centro Oeste (UNICENTRO), Paraná, Brasil. \\ * Autor para correspondência (farinha@ unicentro.br) \\ Recibido: 08/03/2016; Aceptado: 12/04/2016. \\ Doi:10.18004/investig.agrar.2016.junio.37-43
}

\section{RESUMO}

O estudo teve como objetivo avaliar comparativamente a composição florística, diversidade, distribuição espacial e estrutura horizontal das espécies arbóreas em duas áreas distintas, identificadas por A e B, de Savana Estépica Parque, localizada no Parque Estadual do Espinilho, extremo sudoeste do Estado do Rio Grande do Sul, Brasil. Para as análises foram utilizados dados oriundos de duas unidades permanentes de um hectare cada, subdivididas em cem subunidades de cem metros quadrados $(10 \times 10$ $\mathrm{m})$. Foram avaliadas todas as árvores com diâmetro a altura do peito (DAP) igual ou maior que dez centímetros. A estrutura horizontal foi avaliada pelos cálculos das estimativas dos parâmetros fitossociológicos: densidade, dominância, frequência. A riqueza e a diversidade das áreas foram calculadas pelo índice de diversidade de Shannon (H'). Para a análise da similaridade florística entre as duas áreas foi utilizado o índice de similaridade de Sorensen, enquanto que a distribuição espacial das espécies foi analisada por meio do índice de dispersão de Morisita Padronizado. A análise das duas áreas demonstra que o Parque do Espinilho é composto por diferentes tipologias, que resultam em diferenças nas estruturas horizontais, diversidades e no padrão de distribuição espacial, sendo Prosopis affinis a espécie dominante na área $\mathrm{A}$, enquanto que na área $\mathrm{B}$, a dominância foi da espécie Prosopis nigra.

Palavras-chave: dispersão espacial, diversidade florística, fitossociologia, índice de similaridade, Savana Estépica Parque.

\begin{abstract}
The study aimed at comparing the floral composition, diversity, spatial distribution and horizontal structure of tree species in two different areas, identified as " $A$ " and "B", Park Steppe Savannah, located in the Espinilho state Park, southwestern end of the Rio Grande do Sul State, Brazil. The study was carried out using data from two standing units, one hectare each, divided into one hundred subunits measuring one hundred square meters $(10 \mathrm{x} 10$ m) each. Trees with a diameter of $10 \mathrm{~cm}$ or above $10 \mathrm{~cm}$ at breast height $(\mathrm{DBH})$ were evaluated. Horizontal structure was evaluated by phytosociological parameters: abundance, dominance, frequency. The richness and diversity of the areas were calculated by the Shannon diversity index $\left(\mathrm{H}^{\prime}\right)$. Sorensen similarity index was used for the analysis of floristic similarity between the two areas, whereas spatial dispersion of species was analyzed by the Morisita Standardized dispersion index. The comparison between the two areas showed that Espinilho State Park is made up of different types of vegetation, resulting in differences in horizontal structures, diversity and spatial distribution pattern, being Prosopis affinis, the dominant species in area A whereas in area B, Prosopis nigra was the dominant one.
\end{abstract}

Key words: Spatial dispersion, floristic diversity, phytosociology, similarity index, Steppe Savannah Park. 


\section{INTRODUÇÃO}

De acordo com o Inventário Florestal Contínuo (2010), o Estado do Rio Grande do Sul é contemplado com dois biomas: a Mata Atlântica e o Pampa, subdivididos em nove regiões fitogeográficas, as quais destacam a Floresta Ombrófila Mista, a Floresta Estacional Decidual, a Floresta Estacional Semidecidual e a Estepe GramíneoLenhosa, além das regiões de contato entre estas formações, que formam ecossistemas totalmente distintos, com alta riqueza de espécies.

No extremo sudoeste do estado do Rio Grande do Sul, no município de Barra do Quaraí, entroncamento do Rio homônimo com o Rio Uruguai, na tríplice divisa com Brasil, Argentina e Uruguai, ocorre uma formação vegetal de características únicas, denominada de Savana Estépica Parque, que recobre totalmente uma área denominada Parque do Espinilho (Marchiori et al. 2014).

A bibliografia disponível sobre a formação vegetal encontrada no Parque do Espinilho é relativamente escassa. $\mathrm{O}$ primeiro autor a pesquisar as características endêmicas da vegetação encontrada no Parque do Espinilho foi Rambo em 1942. A partir daí outros estudos e aprofundamentos se seguiram, com destaque para Marchiori e colaboradores. Dentre os trabalhos mais representativos pode-se citar Rambo (1942); Marchiori et al. (1985); Simas et al. (2002); Galvani e Baptista (2003); Marchiori (2002); Simas et al. (2002); Marchiori, (2004); Watzlawick et al. (2010); Alves e Marchiori, (2011); Redin et al. (2011); Marchiori et al. (2014); Watzlawick et al. (2014).

Esta formação vegetal campestre apresenta características fisionômicas típicas, com arbustos e pequenas árvores em geral de mesma espécie, com distribuição bastante espaçada, semelhantes a florestas plantadas. Apresenta-se como uma pseudo-ordenação de plantas lenhosas sobre denso tapete, principalmente de plantas herbáceas e gramíneas, que conferem a estepe um caráter de Parque. A Savana-Estépica Parque geralmente recobre pequenas depressões capeadas por vertissolos, que na época das chuvas, são alagadas por não possuírem boa drenagem (IBGE 2012). O conceito geral da Savana Estépica está associado ao xeromorfismo, manifestado, por meio da abundância de vegetais decíduos e espinhosos, como cactáceas e bromeliácea e pela estacionalidade da vegetação herbáceo-graminóide que, durante o período desfavorável, perde suas folhas (Leite 2002).
As espécies de porte arbustivo-arbóreo mais comuns encontradas na Savana Estépica Parque nessa latitude, segundo Marchiori et al. (1983, 1985) são: algarrobo (Prosopis nigra (Griseb.) Hieron) e inhanduvá (Prosopis affinis Spreng.), quebracho-blanco (Aspidosperma quebracho-blanco $\quad$ Schltdl.), quebracho-flojo (Acanthosyris spinescens (Mart. \& Eichler) Griseb.), em geral, espinhosas e decíduas, como também o espinilho (Vachellia caven (Molina) Molina) a coronilla (Scutia buxifolia Reissek), elementos arbóreos peculiares no Parque Espinilho típico.

Simas et al. (2002) definem a fisionomia vegetal do Parque do Espinilho como um local composto por árvores pequenas, isoladas ou em pequenos grupos, cujas copas não se sobrepõem, possibilitando o crescimento de gramíneas, o que permite ao mesmo tempo o pastoreio e o sombreamento como proteção para o gado, sendo esta atividade, um dos principais fatores de alteração da dinâmica, prejudicando a regeneração natural das espécies arbóreas do local.

O aspecto fitofisionômico no Parque do Espinilho, segundo Reitz et al. (1983) é formado principalmente pela associação das duas espécies do gênero Prosopis, concentradas principalmente nos arredores da Barra do Quaraí-RS, constituindo-se de 90 a $98 \%$ da vegetação arbórea do parque. Pode-se encontrar também com relativa frequência o espinilho (Vachellia caven), dando à esta vegetação o aspecto xeromórfico típico do local.

Esta tipologia abriga uma vegetação peculiar, que não é encontrada em outras áreas do país, com presença de espécies endêmicas e vulneráveis, bem como variações espaciais em sua composição e estrutura, ocorridas devido condições naturais e antrópicas. Segundo Watzlawick et al. (2010), o local vem sofrendo com a intensa alteração antrópica, devido à mudança do uso da terra, na qual a vegetação nativa é substituída por pastagens, para o cultivo de grãos, ou mesmo utilizada para o pastoreio de forma intensiva, reduzindo assim a área com vegetação típica de Savana Estépica Parque.

Diante a estes fatores, o maior conhecimento sobre as características desta formação vegetal e a identificação de associações ou unidades tipológicas dentro do Parque do Espinilho é importante para conservação e manejo apropriado. Por esta razão, o presente estudo teve como objetivo verificar as possíveis diferenças na estrutura e composição florística da Savana Estépica Parque, em 
locais distintos do Parque Estadual do Espinilho, RS, Brasil.

\section{MATERIAL E MÉTODOS}

\section{Área de estudo}

As áreas de estudo estão localizadas no Parque Estadual do Espinilho, no município de Barra do Quaraí, no extremo sudoeste do estado do Rio Grande do Sul, Brasil, conforme Figura 1, situado entre as coordenadas geográficas $449097 \mathrm{~m}$ e $455853 \mathrm{~m}$ de longitude Leste e $6659840 \mathrm{~m}$ e $6659215 \mathrm{~m}$ de latitude Sul, a uma altitude média de 52 m. O Parque Estadual do Espinilho está inserido na microbacia do Arroio Quaraí-Chico, possuindo uma área de 1.617,14 ha, conforme Decreto Estadual 41.444 de 28 de fevereiro de 2002.

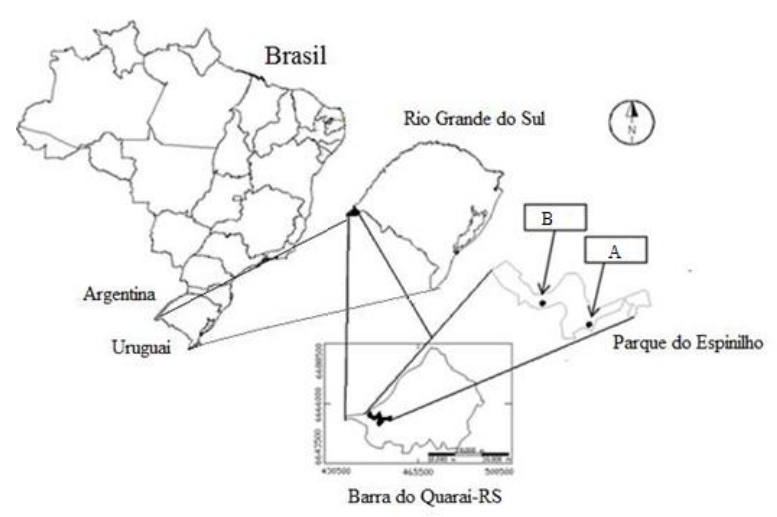

Figura 1. Área de estudo e localização das unidades amostrais A e B.

O clima da região é do tipo $C f a$ ou subtropical úmido com verão quente, conforme a classificação climática de Köppen-Geiger, com temperaturas médias anuais em torno de $19,4^{\circ} \mathrm{C}$, porém com ocorrência frequentes de geadas no inverno. As chuvas são bem distribuídas durante todo o ano, com uma média anual variando entre 1.300 e $1.500 \mathrm{~mm}$, apresentando índices médios mensais superiores a $100 \mathrm{~mm}$, o que não exclui a ocorrência de déficits hídricos nos meses mais quentes (Almeida 1996). O solo é do tipo Chernossolo, moderadamente drenado, de coloração bruno-escura ou bruna-acinzentada, derivados de diferentes litologias (EMBRAPA 2013).

A vegetação natural, conforme classificação do IBGE (2012), pertence à região da Savana-Estépica Parque, a qual está associada ao xerofitismo, apresentando características fisionômicas muito típicas, com arbustos e pequenas árvores, em geral de mesma espécie, e distribuição bastante espaçada.
Para a análise florística e fitossociológica utilizou-se dados oriundos de medições realizadas em 2009 em duas unidades permanentes de um hectare cada (100 x $100 \mathrm{~m})$, identificadas como unidades A e $\mathrm{B}$, respectivamente, distantes 6,8 km uma da outra (Figura 1), subdivididas em cem subunidades de cem metros quadrados (10 x $10 \mathrm{~m})$. As avaliações foram realizadas em todas as árvores com diâmetro a altura do peito (DAP) igual ou maior que dez centímetros com auxílio de fita métrica, as quais foram mensuradas as alturas totais com a utilização do clinômetro Hagöf, para posterior cálculo das alturas máximas, médias e mínimas.

A identificação taxonômica foi realizada inicialmente in loco, bem como utilizado consultas ao Herbário do Departamento de Ciências Florestais (HDCF) da Universidade Federal de Santa Maria - Rio Grande do Sul, Brasil. A elaboração da lista da composição florística foi organizada de acordo com a Angiosperm Phylogeny Group (APG III 2009).

A estrutura horizontal foi avaliada pelos cálculos das estimativas dos parâmetros fitossociológicos tradicionalmente utilizados: densidade, dominância, frequência, em valores absolutos e relativos, e o valor de importância, conforme metodologia de Mueller-Dumbois e Ellenberg (1974).

Para melhor entender a riqueza e a diversidade das áreas foram calculados os índices de diversidade de Shannon $\left(H^{\prime}\right)$, sendo testada a diferença estatística entre a diversidade entre os dois locais através da metodologia de Hutchenson (1970). Também foi verificada a similaridade florística entre as duas áreas pelo Índice de Similaridade de Sorensen (Brower e Zar 1984), enquanto que a dispersão espacial das espécies foi analisada por meio do índice de dispersão de Morisita Padronizado (Krebs 1999).

As estimativas dos parâmetros fitossociológicos, índice de diversidade, foram calculados por meio do software SADEF (Gardin 2011). A análise da dispersão espacial através do software $R$ Statistic e os demais cálculos, com o uso da planilha eletrônica Microsoft Excel 2013®.

\section{RESULTADOS Y DISCUSSÃO}

\section{Florística, similaridade e diversidade de espécies}

Na unidade permanente A foram encontradas apenas quatro espécies: Prosopis affinis Spreng. (Fabaceae), 
Prosopis nigra (Griseb.) Hieron (Fabaceae), Vachellia caven (Molina) Seigler \& Ebinger (Fabaceae) e Scutia buxifolia Reissek (Rhamnaceae), enquanto que na unidade B, ocorreram o dobro de espécies, sendo estas: Aspidosperma quebracho-blanco Schltdl. (Apocynaceae), Chrysophyllum marginatum (Hook. e Arn.) Radlk. (Sapotaceae), Myrcia selloi (Spreng.) N. Silveira (Myrtaceae), Prosopis affinis, Prosopis nigra, Vachellia caven (Fabaceae), Sapium haematospermum Müll. Arg. (Euphorbiaceae) e Scutia buxifolia (Rhamnaceae).

Considerando a divisão proposta por Marchiori et al. (2014), em que o Parque do Espinilho pode ser dividido em três unidades tipológicas distintas de vegetação: Mata ciliar, Parque de Inhanduvá e Parque de Algarrobos, a unidade permanente A pode ser enquadrada como parte do Parque de Inhanduvá, devido à predominância de Prosopis affinis, chamada popularmente de Inhanduvá, e a unidade $\mathrm{B}$ pertencente à região chamada de Parque de Algarrobos, devido à predominância de Prosopis nigra, chamada popularmente de Algarrobo (Tabela 1 e 2).

A análise da diversidade florística das duas áreas, analisada pelo Índice de Shannon (H'), apresentou para a unidade A o valor de diversidade de 0,72 nats/indivíduo e a unidade $\mathrm{B}$ o valor de 1,51 nats/indivíduo, demonstrando haver nítidas diferenças entre as áreas. A diferença de diversidade de espécies é comprovada pelo Teste de Hutchenson (Hutchenson 1970), que revelou diferença estatística entre os dois locais a $1 \%$ de probabilidade.

A similaridade florística entre as duas áreas, calculadas através do índice de Similaridade de Sorensen foi de $66,6 \%$ (Figura 2), considerada alta de acordo com Kent e
Coker (1992), com quatro espécies ocorrendo em comum entre as duas áreas (Prosopis affinis, Prosopis nigra, e Vachellia caven e Scutia buxifolia) e quatro exclusivas da unidade B (Aspidosperma quebracho-blanco, Chrysophyllum marginatum, Myrcia selloi e Sapium haematospermum).

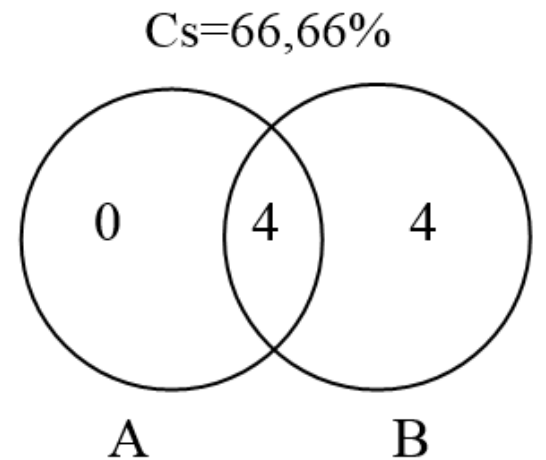

Figura 2. Diagrama de Venn para as duas unidades amostrais A e B e as espécies que ocorrem de maneira compartilhada ou exclusiva entre elas.

\section{Fitossociologia}

Na unidade A, a espécie do gênero Prosopis affinis é considerada a de maior importância (Tabela 1), sendo também a de maior densidade, área basal e frequência, seguida de Prosopis nigra e Vachellia caven e apenas um indivíduo de Scutia buxifolia.

Tabela 1. Índices fitossociológicos da estrutura horizontal, vertical e distribuição espacial da vegetação da unidade A

\begin{tabular}{lccccccccc}
\hline \multicolumn{1}{c}{ Nome Científico } & $\begin{array}{c}\text { DA } \\
(\mathbf{N} / \mathbf{h a})\end{array}$ & $\begin{array}{c}\text { DR } \\
(\boldsymbol{\%})\end{array}$ & $\begin{array}{c}\text { DoA } \\
\left(\mathbf{m}^{2} / \mathbf{h a}\right)\end{array}$ & $\begin{array}{c}\text { DoR } \\
(\boldsymbol{\%})\end{array}$ & $\begin{array}{c}\text { FA } \\
(\mathbf{N})\end{array}$ & $\begin{array}{c}\text { FR } \\
(\boldsymbol{\%})\end{array}$ & VI & IMP & Class. IMP \\
\hline Prosopis affinis & 107,0 & 75,9 & 3,48 & 82,5 & 43,0 & 71,7 & 76,7 & 0,51 & Agregada \\
Prosopis nigra & 24,0 & 17,0 & 0,61 & 14,6 & 11,0 & 18,3 & 16,6 & 0,53 & Agregada \\
Scutia buxifolia & 1,0 & 0,7 & 0,01 & 0,3 & 1,0 & 1,7 & 0,9 & 0,00 & NA \\
Vachellia caven & 9,0 & 6,4 & 0,11 & 2,6 & 5,0 & 8,3 & 5,8 & 0,56 & Agregada \\
\hline \multicolumn{1}{c}{ TOTAL } & 141 & - & 4,218 & - & - & - & - & - & - \\
\hline
\end{tabular}

Onde: $\mathrm{DA}=$ Densidade Absoluta; DR = Densidade Relativa; DoA= Dominância absoluta; DoR= Dominância relativa; $\mathrm{FA}=$ Frequência absoluta; FR = Frequência Relativa; VI = Valor de Importância; IMP= Índice de Dispersão de Morisita Padronizado; Class. IMP: Classificação da distribuição da espécie conforme o Índice de Dispersão de Morisita Padronizado.

Na unidade B, Tabela 2, a espécie Prosopis nigra é a mais importante e a mais frequente dentro das subunidades, além de também ter grande densidade populacional. $\mathrm{O}$ Aspidosperma quebracho-blanco também apresenta alta ocorrência, sendo a segunda espécie de maior valor de importância, seguida de Scutia buxifolia. Também foram encontrados cinco indivíduos mortos no local. 
Tabela 2. Índices fitossociológicos da estrutura horizontal, vertical e distribuição espacial da vegetação da unidade B.

\begin{tabular}{lccccccccc}
\hline \multicolumn{1}{c}{ Nome Científico } & $\begin{array}{c}\text { DA } \\
(\mathbf{N} / \mathbf{h a})\end{array}$ & $\begin{array}{c}\text { DR } \\
(\boldsymbol{\%})\end{array}$ & $\begin{array}{c}\text { DoA } \\
(\mathbf{m} / \mathbf{h a})\end{array}$ & $\begin{array}{c}\text { DoR } \\
(\boldsymbol{\%})\end{array}$ & FA & FR & VI & IMP & Class. IMP \\
\hline Prosopis nigra & 74,0 & 47,7 & 1,427 & 38,7 & 28,0 & 41,2 & 42,5 & 0,52 & Agregada \\
Aspidosperma quebracho- & 30,0 & 19,4 & 1,366 & 37 & 14,0 & 20,6 & 25,7 & 0,53 & Agregada \\
blanco & 21,0 & 13,5 & 0,321 & 8,7 & 2,0 & 2,9 & 8,4 & 0,91 & Agregada \\
Scutia buxifolia & 11,0 & 7,1 & 0,109 & 3 & 8,0 & 11,8 & 7,3 & 0,52 & Agregada \\
Vachellia caven & 9,0 & 5,8 & 0,15 & 4,1 & 7,0 & 10,3 & 6,7 & 0,50 & Aleatória \\
Prosopis affinis & 3,0 & 1,9 & 0,026 & 0,7 & 2,0 & 2,9 & 1,9 & 0,60 & Agregada \\
Myrcia selloi & 1,0 & 0,6 & 0,016 & 0,4 & 1,0 & 1,5 & 0,9 & 0,00 & NA \\
Chrysophyllum marginatum & 1,0 & 0,6 & 0,008 & 0,2 & 1,0 & 1,5 & 0,8 & 0,00 & NA \\
Sapium haematospermum & $155^{*}$ & - & 3,689 & - & - & - & - & - & - \\
\hline TOTAL & & & & &
\end{tabular}

Onde: $\mathrm{DA}=$ Densidade Absoluta; DR = Densidade Relativa; DoA= Dominância absoluta; DoR= Dominância relativa; FA= Frequência absoluta; FR = Frequência Relativa; VI = Valor de Importância; IMP= Índice de Dispersão de Morisita Padronizado; Class. IMP: Classificação da distribuição da espécie conforme o IMP. * Incluindo cinco árvores mortas.

Na unidade A, das quatro espécies encontradas, a Prosopis affinis e a Prosopis nigra correspondem a mais de $92,90 \%$ dos indivíduos amostrados, sendo que somente a Prosopis affinis representa $75,9 \%$. As espécies Vachellia caven e a Scutia buxifolia ocupam um papel secundário na composição da estrutura e florística dessa unidade permanente. Em levantamento no Parque do Espinilho realizado por Marchiori et al. (1985), na área chamada de Parque de Inhanduvá, encontraram dados semelhantes a esses, no qual a Prosopis affinis predominava amplamente, representando $70 \%$ das árvores, com 82 indivíduos, e a Prosopis nigra correspondem a mais de 12,07\%, com 14 indivíduos. Os dados apresentados na Tabela 1, corroboram com a afirmação de Reitz et al. (1983) que a Prosopis affinis é a espécie mais importante para a fisionomia e estrutura da vegetação da unidade do Parque de Inhanduvá, sendo a que apresenta os valores mais elevados de abundância, dominância e frequência.

Na unidade $\mathrm{B}$ ocorreu um número maior de espécies, sendo as com maior densidade a Prosopis nigra, Aspidosperma quebracho-blanco, Scutia buxifolia, Vachellia caven e Prosopis affinis, que representam 93,5\% de todas as espécies amostradas. Comparando-se esses valores, com os encontrados por Marchiori et al. (2014) na unidade Parque de Algarrobos, os quais foram 31,56\% para Prosopis nigra, 25,66\% para Vachellia caven e 23,60\% para Aspidosperma quebracho-blanco, verifica-se que a Prosopis nigra, seguida da Aspidosperma quebracho-blanco são as espécies características da unidade Parque de Algarrobos.

Os dados da unidade B corroboram com a afirmação de Marchiori et al (2014) de que além da participação expressiva de Prosopis nigra e Aspidosperma quebrachoblanco, os baixos valores de abundância, dominância e frequência de Prosopis affinis são aspectos fitossociológicos que nitidamente contrastam com os observados na unidade Parque de Inhanduvá.

A unidade A apresentou maior área basal $\left(4,218 \mathrm{~m}^{2} \mathrm{ha}^{-1}\right)$ do que a unidade $\mathrm{B}\left(3,689 \mathrm{~m}^{2} \mathrm{ha}^{-1}\right)$, explicado pelo DAP médio maior na primeira unidade $(18,2 \mathrm{~cm})$ do que na segunda $(15,9 \mathrm{~cm})$, resultante principalmente pela grande densidade de indivíduos de Prosopis affinis de maior diâmetro.

Com relação à altura dos indivíduos desta formação, a unidade A apresentou uma média de $3,5 \mathrm{~m}$, com coeficiente de variação dos indivíduos arbóreos de 21,6\%, enquanto que na unidade $B$, observou-se maior média de altura (4,9 m) e maior coeficiente de variação (34\%), indicando maior variabilidade, tendo a média geral sofrido aumento devido à interferência direta dos indivíduos de Aspidosperma quebracho-blanco e Scutia buxifolia que se apresentaram em maior porte na unidade. 
Com a análise da distribuição espacial dos indivíduos, pelo índice de Morisita Padronizado, constata-se que todas as espécies presentes na área A possuem padrão de distribuição agregado. Para a espécie Scutia buxifolia não foi possível classifica-la, devido a ocorrência de apenas um indivíduo no local.

Em relação à área $\mathrm{B}$, com exceção das espécies Prosopis affinis e dos indivíduos mortos, que apresentaram distribuição aleatória, todas as demais espécies tiveram distribuição agregada.

O padrão de distribuição agregado da maioria das espécies nas duas áreas analisada pode ser justificado pela grande variação no número de indivíduos nas subunidades amostrais. Marchiori et al. (1985) verificam haver grande gregarismo das espécies de Prosopis no Parque do Espinilho. Muitas das subunidades formadoras de ambas as áreas não apresentaram nenhum indivíduo com DAP maior que $10 \mathrm{~cm}$, sendo que apenas $52 \%$ e $67 \%$ das subunidades, respectivamente nas áreas A e B, tiveram árvores amostradas.

A Prosopis affinis, que na área B foram identificados nove indivíduos e apresentou padrão aleatório, encontrase distribuída em nove subunidades. Este aspecto pode estar ligado às interferências antrópicas que ocorrem no local, como retirada de madeira, subtraindo-se os indivíduos adultos e a pecuária, que pode afetar na sua regeneração natural, provocando sua aleatoriedade espacial.

O índice de diversidade Shannon ( $\left.\mathrm{H}^{\prime}\right)$ revelou maior diversidade florística na unidade $\mathrm{B}$, com o valor de 1,51, enquanto que na unidade $\mathrm{A}$ foi de 0,72. O Teste de Hutcheson (1970) para o índice de Shannon (H') apontou que existe diferença estatística significativa entre a diversidade dessas duas unidades. A maior diversidade de espécies encontradas na área B foi influenciada pela ocorrência de espécies que podem ser consideradas raras, com menos de 5 indivíduos por hectare.

O maior valor do índice H' na unidade B ocorre devido a este local possuir o dobro de espécies que as encontradas na área A. Resultado semelhante ao encontrado na unidade B foi observado por Watzlawick et al. (2014), onde em uma área de quatro hectares próxima da unidade B, também pertencente ao Parque do Espinilho, foi encontrado o valor de 1,86. Já Giménez et al. (2007), encontraram o valor de 2,60 no Parque Los Quebrachos, na Argentina, enquanto Ramos (2008), encontrou o valor de 1,88, em Rincón de Franquía, no Uruguai, cuja sinúsia é semelhante ao Parque do Espinilho.

\section{CONCLUSÕES}

A análise das duas áreas localizadas no Parque Estadual do Espinilho, identificadas por A, enquadrada como Parque de Inhanduvá, com predominância da espécie Prosopis affinis e B, como Parque de Algarrobos e predominância de Prosopis nigra, demonstram que o Parque é composto por diferentes tipologias vegetais. $\mathrm{O}$ número de indivíduos levantados na área $\mathrm{B}$ foi $10 \%$ maior em relação a área $\mathrm{A}$ e a similaridade florística entre as duas áreas foi considerada alta, porém com diversidade florística maior na área B, justificada pela ocorrência de espécies raras encontradas.

Nos últimos anos esta formação vegetal vem sofrendo intensas alterações em sua estrutura natural, decorridas principalmente por atividades antrópicas, levando a perda sucessiva de sua cobertura original. A formação vegetal Savana Estépica Parque localizada na Barra do Quaraí é única e exclusiva no Brasil e o maior conhecimento sobre as características naturais e a identificação de suas unidades tipológicas vegetais mostra-se importante para a sua conservação, recuperação e manejo apropriado, sendo necessários mais estudos sobre suas características naturais.

\section{REFERENCIAS BIBLIOGRAFICAS}

Almeida, J. 1996. O solo nos grandes domínios morfoclimáticos do Brasil e desenvolvimento sustentado - Solo dos Pampas. In Alvarez, VHV; Fontes, LEF; Fontes, MPF. Os solos nos grandes domínios morfoclimáticos do Brasil e o desenvolvimento sustentado. Viçosa, BR, SBCS/UFV, $930 \mathrm{p}$.

Alves, FS; Marchiori, JNC. 2011. O Inhanduvá no Rio Grande do Sul: enfoque fitogeográfico. Ciência e Ambiente 42:39-70.

Bremer, B; Bremer, K; Chase, MW. 2009. An update of the Angiosperm Phylogeny Group classification for the orders and families of flowering plants: APG III. Botanical Journal of the Linnean Society 161:105121.

EMBRAPA (Empresa Brasileira de Pesquisa Agropecuária, BR) 1999. Sistema brasileiro de 
classificação de solos. Brasília, BR, EMBRAPA. 412 p.

Galvani, FR; Baptista, LR. 2003. Flora do parque estadual do Espinilho: barra do Quarai/RS. Revista da FZVA $10(1): 42-62$.

Giménez, AM; Hernández, P; Gerez, R; Rios, NA. 2007. Diversidad vegetal ensiete unidades demostrativas del chaco semiárido argentino. Madera y Bosques. 13:6178.

Hutcheson, K. 1970. A test for comparing diversities based on the Shannon formula. Journal of theoretical Biology. 29(1):151-154.

IBGE (Instituto Brasileiro de Geografia e Estatística, BR). 2012. Manual técnico da vegetação brasileira. Rio de Janeiro, BR, IBGE. 271 p.

Kent, MC; Coker, PP. 1992. Vegetation description and analysis: a practical approach. London, UK, British Library. 363 p.

Leite, FP. 2002. Contribuição ao conhecimento fitoecológico do sul do Brasil. Ciência \& Ambiente 24:51-73.

Marchiori, JNC; Longhi, SJ; Galvão, L. 1983. O gênero Prosopis L. (Leguminosae Mimosoideae) no Rio Grande do Sul. Ciência e Natura 5:171-177.

Marchiori, JNC; Longhi, SJ; Galvão, L. 1985. Composição florística e estrutura do parque de inhanduvá no Rio Grande do Sul. Revista Centro de Ciências Rurais 15(4):319-334.

Marchiori, JNC. 2002. Fitogeografia do Rio Grande do Sul: enfoque histórico e sistemas de classificação. Porto Alegre, BR, EST. 118 p.

Marchiori, JNC. 2004. Fitogeografia do Rio Grande do Sul: Campos Sulinos. Porto Alegre, BR, EST. 110 p.

Marchiori, JNC; Alves, FS; Deble, LP; Silveira de Oliveira-Deble, A. 2014. Vegetação do Parque Estadual do Espinilho: origem do nome e considerações fitogeográficas. Balduinia 44:01-16.
Moreno, JA. 1961. Clima do Rio Grande do Sul. Porto Alegre, BR, Governo do Estado do Rio Grande do Sul / Secretaria da Agricultura. 41 p.

Rambo, B. 1942. A Fisionomia do Rio Grande do Sul: ensaio de monografia natural. Porto Alegre, BR, Impr. Oficial. 456 p.

Ramos, G. 2008. Florísitca y fitosociología preliminarde la vegetación nativa leñosa de Rincón de Frabquía. Tesis Tec. Bella Unión, UY, FCIEN/UDELAR. 49 p.

Redin, CG; Longhi, RV; Watzlawick, LF; Longhi, SJ. 2011. Composição florística e estrutura da regeneração natural do Parque Estadual do Espinilho, RS. Ciência Rural 41(7):1195-1201.

Reitz, R; Klein, RM; Reis, A. 1983. Projeto Madeira do Rio Grande do Sul: anais botânicos do herbário Barbosa Rodrigues. Sellowia 34/35(34/35):5-483.

Simas, VR; Costa, EC; Aires Simas, C. 2002. Vegetação arbórea fanerógama ocorrente em área de nidificação de Atta vollenweideri (Forel, 1983) (Hymenoptera: formicidae). Revista da Faculdade de Zootecnia, Veterinária e Agronomia 9:79-88.

UFSM (Universidade Federal de Santa Maria, BR). 2010. Inventário Florestal Contínuo: base de datos (en línea). Santa Maria, BR. Consultado 13 jan. 2015. Disponível em http://coralx.ufsm.br/ifcrs/index.php.

Watzlawick, LF, Longhi, SJ; Schneider, PR; Finger, CAG; Longhi, RV. 2010. Caracterização e dinâmica da vegetação de uma Savana Estépica Parque, Barra do Quaraí-RS/Brasil. Pesquisa Florestal Brasileira 30(64):363-368.

Watzlawick, LF; Longhi, SJ; Schneider, PR; Finger, CAG. 2014. Aspectos da vegetação arbórea em fragmento de Estepe Estacional Savanícola, Barra do Quaraí-RS, Brasil. Ciência Florestal. 24(1):23-36. 